\section{Induced Radioactivity of Fluorine and Calcium}

IN an earlier paper ${ }^{1}$, I have shown that sodium and phosphorus become radioactive under bombardment with alpha rays, presumably corresponding to the creation of $\mathrm{Al}^{26}$ and $\mathrm{Cl}^{34}$ respectively. In the case of phosphorus, chemical separation of the active body, kindly carried out by Prof. S. Sugden, gave strong support to this picture. Between these two unstable isotopes lies a third one, $\mathrm{P}^{30}$, found by Curie and Joliot ${ }^{2}$. It was pointed out that another link of that chain, $\mathrm{Na}^{22}$, should be produced by bombarding fluorine with alpha rays. Since the periods of the three above-mentioned bodies decrease rapidiy with atomic number, a very short period was anticipated for $\mathrm{Na}^{22}$. The search for this substance was unsuccessful, however, although periods so short as one tenth of a second would have been detectable by the method applied.

About that time, the emission of positrons from fluorine under alpha-ray bombardment was reported in a paper by L. Meitner ${ }^{3}$. The positrons were observed only during bombardment, and no life period was given. This was generally taken as evidence for a very short period. However, the very low energy of the positrons, as measured by Meitner, rather seemed to point to a long period. On inquiry, I was kindly informed by Prof. Meitner that no attenupt at all had been made to measure the period, simply because the experiments had been carried out before the discovery of induced radioactivity. I have therefore recently taken up the search for an isotope $\mathrm{Na}^{22}$ of long period.

Through the courtesy of the Committee of the Radium Institute and Finsen Hospital, I was enabled to use radon in quantities up to 600 millicuries as the source of alpha rays. The radon was put into small glass vessels with a thin mica window which reduced the range to about $6 \mathrm{~cm}$. Upon bombarding calcium fluoride, fairly strong effects were obtained at once; other calcium compounds, however, gave similar effects. The search for $\mathrm{Na}^{22}$ was therefore continued with sodium fluoride and lithium fluoride. In both cases weak activity was observed after prolonged bombardment. A chemical separation, kindly carried out by Prof. G. von Hevesy, showed that the active body follows the reactions of sodium, and therefore is presumably $\mathrm{Na}^{22}$.

In striking contrast with the first anticipations, the outstanding feature of this new activity is its long period. One sample has been under observation for three weeks, with no evidence of decay. It may be concluded that the half-period must be at least six months. This explains the apparent weakness of the effect; indeed, with a few days' bombardment, only a fraction of the equilibrium activity is obtained. On the other hand, a reasonable assumption about the equilibrium activity leads to an estimated life of from one to several years.

The particles emitted from $\mathrm{Na}^{22}$ were shown by magnetic deflection to be positrons. They are rapidly absorbed in aluminium, the half-value thickness being about $0.03 \mathrm{gm} . / \mathrm{cm} .^{2}$. This corresponds to a mean energy of about $2 \times 10^{5}$ electron-volts, in good agreement with the results of Meitner.

The activity produced in calcium has also been studied. The half-period is $\mathbf{4} \cdot \mathbf{4}$ hours, with a possible error of ten per cent. The particles emitted are positrons, and their half-value thickness of aluminium is about $0.06 \mathrm{gm} . / \mathrm{cm} .^{2}$. From the great intensity, one may say that the effect is due to the main isotope of calcium, $\mathrm{Ca}^{40}$. Capture of the alpha particle, with subsequent emission of a proton or neutron, would lead to the formation of $\mathrm{Sc}^{43}$ or $\mathrm{Ti}^{43}$, respectively. A chemical separation, kindly carried out by Prof. G. von Hevesy, showed that the active body follows the reactions of scandium. Therefore the $4 \cdot 4$ hours activity certainly corresponds to $\mathrm{Sc}^{43}$. Of course, it cannot be excluded that in the first instance $\mathrm{Ti}^{43}$ may be formed, and quickly transformed into $\mathrm{Sc}^{43}$ by emission of a positron. No short-period activity, however, could be detected.

\section{Institute for Theoretical Physics, Copenhagen. July 3.}

I NATURE, 133, 712; 1934.
NATURE, 133, $201 ; 1934$.
3 Naturwis8., 22, $420 ; 1934$.

\section{The Rate of Photosynthesis}

THE recent correspondence on photosynthetic rates ${ }^{1,2,3}$ has emphasised numerous significant facts now known about the process. A few important ones still remain. They can be roughly summarised by saying that the equations proposed seem to contain no term for the plant.

In the proposals of Burk and Lineweaver ${ }^{2}$ the symbol $\left(\mathrm{CO}_{2}\right)$ is used without qualification, and the authors say that their equation provides for hyperbolic relations between $y$ (rate) and $\left(\mathrm{CO}_{2}\right)$ "in accordance with experiment". Presumably, therefore, $\left(\mathrm{CO}_{2}\right)$ is to be read as the external and measurable concentration of carbon dioxide. But this cannot be the effective concentration in their reaction (I)

$$
\mathrm{Chl}+\mathrm{CO}_{2} \rightleftharpoons \text { complex. }
$$

I have been able to show that the rate of photosynthesis can be made to vary considerably when all other conditions are kept constant and only the method of supply of carbon dioxide varied. Shortening the external diffusion path increases the rate, but there always remains the distance (measurable in $\mu$ ) between the outer cell surface and the chloroplast. With moderate carbon dioxide concentrations and intensities of light, this is never negligible ${ }^{5}$; moreover, even the conception of a constant concentration right up to the cell surface is entirely 'ideal' and not realised with any plant. Allowing for these conditions, the equation

$$
C_{e}=\frac{R K^{\prime}}{A-R}+d R
$$

will give a close fit to the observed rates, where $C_{\boldsymbol{e}}$ is the initial external concentration; $R$ the rate; $K^{\prime}$ the dissociation constant of the chlorophyll- $\mathrm{CO}_{2}$ complex; $A$, a constant independent of $C_{e}$, but varying with light, and $d$ a resistance term including, but probably not solely depending on, the resistance to hydro-diffusion. The full derivation of this equation I have already given elsewhere ${ }^{6}$.

A further difficulty resulting from structural considerations occurs in the absorption of light. A linear relation between light intensity and rate of the primary photochemical reaction may be a permissible approximation in simple cases; but it is more doubtful whether we are entitled to carry over this assumption to a wide range of plants with their exceedingly complex light-absorbing surfaces. We are here also faced with a complication, first 